\title{
Dose Analysis of In Vitro and In Vivo Test for Boron Neutron Capture Therapy (BNCT)
}

\author{
Hamidatul Faqqiyyah ${ }^{1, *}$, Sunarno ${ }^{1}$, Isa Akhlis ${ }^{1}$, and Yohannes Sardjono ${ }^{2}$ \\ ${ }^{1}$ Department of Physics, Faculty of Mathematics and Natural Sciences, Universitas Negeri Semarang, Semarang 50229, Indonesia \\ ${ }^{2}$ Center of Accelerator Science and Technology, National Nuclear Energy Agency, Yogyakarta 55281, Indonesia \\ ${ }^{*}$ Corresponding authors: hamidatulfaqqiyyah@gmail.com
}

KEYWORDS

BNCT

BNCT dose

SHIELD-HIT12A
ABSTRACT The purpose of this study was to determine the in vitro and in vivo doses of boron neutron capture cancer therapy (BNCT) using the SHIELD-HIT12A program. To be able to determine the recoil energy, the research was conducted using the Monte Carlo method. Running data obtained the value of ionization activity and recoil lost. The results showed that in vitro and in vivo doses of BNCT for soft tissue irradiation had a value of $0.312 \times 10^{-2} \mathrm{~Sv}$, which is safe and does not harm healthy body tissue around the cancer cells because it is below the threshold of 1.5 Rem or $15 \times 10^{-3} \mathrm{~Sv}$, in accordance with the provisions of the upper value permitted by the International Commission on Radiation Protection in 1966. While the comparative targets are water, the optimal target absorption dose was obtained at concentrations of $3.232 \times 10^{-3} \mathrm{~Gy}$. The dose of carbon equivalent in water with the type of thermal neutron radiation was $16.16 \times 10^{-3} \mathrm{~Sv}$; this dose is classified as unsafe.

(c) The Author(s) 2018. This article is distributed under a Creative Commons Attribution-ShareAlike 4.0 International license.

\section{INTRODUCTION}

The global death toll from cancer in 2007-2030 is projected to rise $45 \%$ (from 7.9 million to 11.5 million deaths) because of the global population and population aging. New cases of cancer in the same period are expected to increase from 11.3 million in 2007 to 15.5 million by 2030 . Of all cases of cancer, more than half occur in small and developing countries. The most common therapy used in cancer treatment is radiotherapy. One part of radiotherapy that has potential to be developed is boron neutron capture therapy (BNCT).

According to (Sauerwein et al. 2012), BNCT is a binary form of radiation therapy using non-radioactive Boron-10 nuclides to capture thermal neutrons that produce a fast nuclear reaction of ${ }^{10} \mathrm{~B}(\mathrm{n}, \alpha){ }^{7} \mathrm{Li}$. The product of this reaction has a linear characteristic of high energy transfer

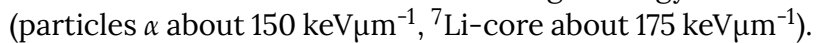
The path length of the particle is in the range of $4.5 \mu \mathrm{m}$ to $10 \mu \mathrm{m}$ resulting in a finite energy deposition to the diameter of a single cell. Theoretically, it is, therefore, possible to selectively irradiate those tumor cells that have taken an adequate amount of ${ }^{10} \mathrm{~B}$. According to Barth et al. (2004), BNCT has a significant advantage with rapid neutron therapy, since alpha and nuclei ${ }^{7} \mathrm{Li}$ particles are selectively deposited. In contrast, neutrons are rapidly delivered non-

$$
\begin{aligned}
& {\left[{ }_{5}^{10} \mathrm{~B}\right]+\left[{ }_{0}^{1} n\right] \longrightarrow\left[{ }_{5}^{11} \mathrm{~B}\right]^{*}-\longrightarrow \begin{array}{l}
{\left[{ }_{2}^{4} \mathrm{He}\right]+\left[{ }_{3}^{7} \mathrm{Li}\right]+2.79 \mathrm{MeV}(6.1 \%)} \\
\longrightarrow\left[{ }_{2}^{4} \mathrm{He}\right]+\left[{ }_{3}^{7} \mathrm{Li}\right]^{*}+2.31 \mathrm{MeV}(93.9 \%)
\end{array}} \\
& \rightarrow\left[{ }_{3}^{7} \mathrm{Li}\right]+\gamma(0.48 \mathrm{MeV})
\end{aligned}
$$

FIGURE 1. Basis of the nuclear reactions according to Barth et al. (2004). selectively to normal tumors and tissues. Figure 1 provides the basis for the nuclear reactions.

Rasouli and Masoudi (2012)'s results show that BNCT is an effective method for destroying brain tumor cells. The recommended epithermal neutron flux for therapy is 109 $\mathrm{n} / \mathrm{cm}^{2} \mathrm{~s}$, which has the most profound effect on the tumor. Files designed for BNCT should include "quality" and "intensity" matches. It is evaluated by two sets of International Atomic Energy Agency (IAEA) recommended values of parameters in the air and in fantasy.

The dose study in BNCT also determines the level of success in cancer therapy. Monte Carlo-based computer codes such as MCNP, MCNPX, PHITS, PENELOPE, GEANT4, FLUKA, EGSnrc, EGS4, BEAMnrc, and SHIELD-HIT12A are required as simulators (Figure 2).

SHIELD-HIT12A is software designed to simulate precision of proton and ion beam proteins in a biological tis-

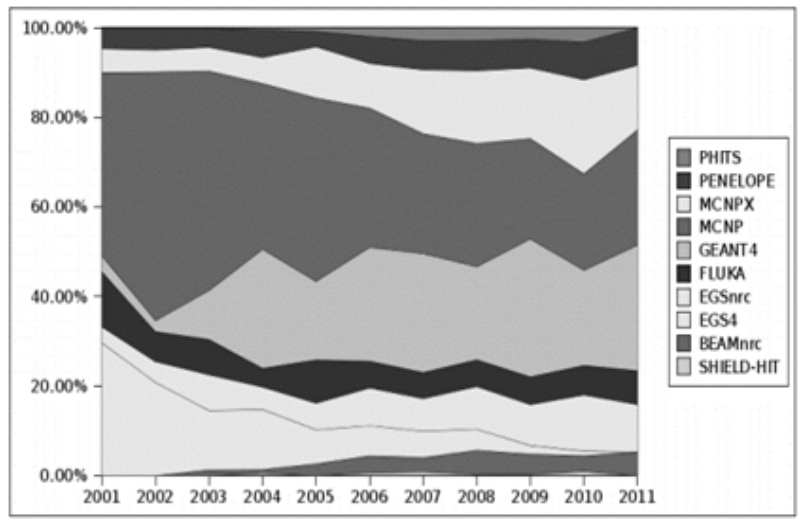

FIGURE 2. Graph of Monte Carlo code usage (Matsumura et al. 2013). 
sue normally used in ion beam cancer therapy. The Monte Carlo particle transport code is for heavy ions that are optimized for particle therapy research. SHIELD-HIT12A is a development of SHIELD-HIT08 and SHIELD-HIT10 which is a massive overhaul of the source code.

In a study conducted by Solleh et al. (2011), the geometry of the reactor core, thermal column, collimator and shield system for BNCT applications REACTOR TRIGA MARK II is simulated with MCNP code. All geometry can be seen in visual editors of MCNP and dosage of neutron particles can be calculated with MCNPX code. But with the limited management and limitations of the MCNP system for dosing calculations at BNCT, the Aarhus Particle Therapy Group developed the SHIELD-HIT12A (Heavy Ion Therapy) program. In Indonesia alone, the study of this program is scarce and still in the early stages so that researchers have a great opportunity to study and develop it further.

\section{MATERIALS AND METHODS}

The research of dose analysis on in vitro and in vivo testing of BNCT was carried out in the Computer Physics Laboratory Faculty of Mathematics and the Natural Sciences, the State University of Semarang, Indonesia.

The tools used in the study comprised hardware in the form of one laptop computer (with the following specifications: ASUS model X455L; Windows 7 Ultimate 64-bit operating system; Intel Core i3-4030U CPU @ 1.90 GHz; and 3.89 GB RAM) and software in the form of SHIELD-HIT12A v0.3.0, Command Prompt (cmd), and finally SimpleGeo 4.3 for visualizing geometries.

To obtain the data on a Windows system, first we ran SHIELD-HIT12A by finding a sample program in the base directory. The subsequent procedure followed the basic required to produce the desired output files. Windows should open a shell (Run- "Enter a command" - cmd) then enter \$ shield hit examples simple. After SHIELD-HIT12A stops running, some new files can be found in the directory. The results from the detector are defined in detect.dat and are stored in the files ex_zmsg.bdo, ex_ymsh.bdo, ex_yzmsh.bdo and ex_cyl.bdo. These files are in binary form and the bdo2txt script can be used to convert them to ASCII format. The next step up to the directory and apply \$ bdo2txt ex_zmsh.bdo, which will generate the output of a set.out file that can be plotted.

The outcome of running SHIELD-HIT12A, which resulted in the distribution of recoil and ion energy lost within the target, was presented using Microsoft Excel. Further data were processed using the Origin8 program, which displays the results in graphical form. After analyzing the ionization energy distribution relationship and recoil activity lost on the target was graphed. For geometric visualization, SimpleGeo 4.3 was used. SimpleGeo is an interactive modeler that allows for the flexible and easy creation of a model using a drag-and-drop interface, as well as on-the-fly inspection to illustrate the field in the form of a 3-D inversion.

\section{RESULTS AND DISCUSSION}

\subsection{Recoil neutron reaction}

The neutron scattering dose is derived from thermal, epithermal, and rapid neutron scattering. The dose component can be calculated using the SHIELD-HIT12A program based on the Monte Carlo method. The Monte Carlo method is one of the probabilistic methods. SHIELDHIT12A gets its calculations by simulating each particle and recording some aspects of each particle being simulated. The Monte Carlo method can be used to duplicate theoretical statistical processes (such as the interaction of nuclear particles with materials) and can specifically be used for complex problems that cannot be modeled using deterministic methods.

In general, the simulation on SHIELD-HIT12A is displayed in digital computers because the number of iterations required to describe a phenomenon is usually quite large. The sampling of statistics in the SHIELD-HIT12A program is based on a random number selection. In a particle transport sample, randomly distributed opportunities use particle transport data to determine each stage of the particle interaction.

Figures 3 and 4 illustrate the light-specification data codes fired by selecting elemental ions as primary particles. The random seed is the option card that outlines the specifications of random number seeds. The use of the same seed for an identical simulation will produce the same result. The default value is 89736501 .

JPART0 shows the specification of primary particles. If JPARTO is not specific it will be assumed to be a proton. HIPROJ shows the values of A and $\mathrm{Z}$ of heavy ions. TMAX0 is an optional card that explains the initial energy of the primary particles in $\mathrm{MeV} /$ nucleon and energy scattered in $\mathrm{MeV} /$ nucleon, showing as a standard deviation of the Gaussian distribution $(1 \sigma)$. The default value is 250 $\mathrm{MeV} /$ nucleon and the energy scattered $=0$. NSTAT shows the option card showing the demand specification of the total number of simulated primary particles and the number of primary transport after the requested advanced score has been saved.

Figure 5 shows the target geometry data specification being shot. The simulated target in this study is a cylindrical material (with its center at the point $(0,0,0) \mathrm{cm}$. The height of the cylinder is described by a $30 \mathrm{~cm}$ axis vector and its radius of $10 \mathrm{~cm}$ This cylinder is surrounded by a vacuum medium which does not interact with particles. The second cylinder is re-interspersed with a black hole medium in which the particles that hit the medium will not be integrated and have no trace of the particles.

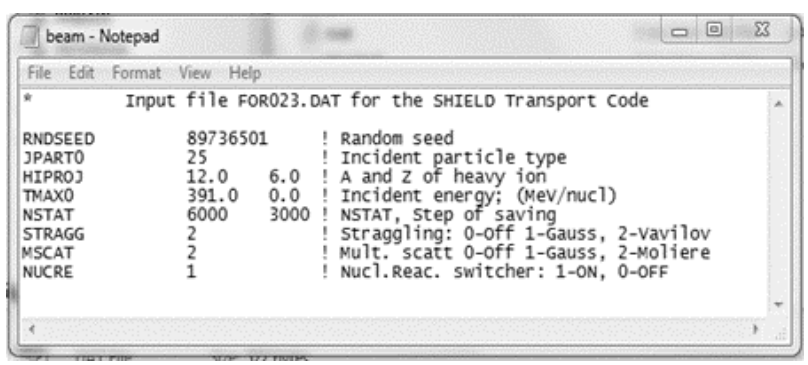

FIGURE 3. Coding the ray specification data is fired by selecting Carbon-12 ions as primary particles.

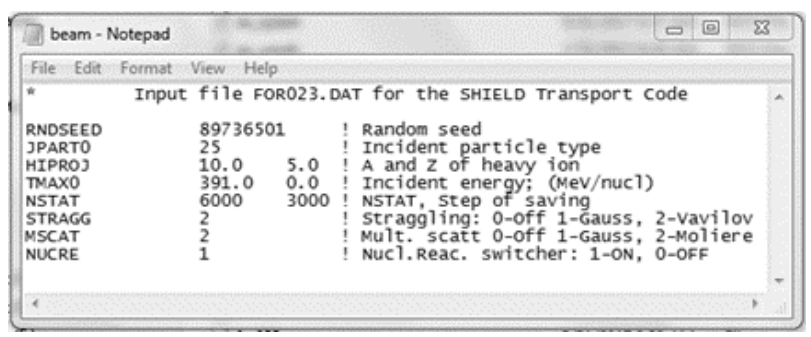

FIGURE 4. Coding the ray specification data is fired by selecting the Boron10 ion as the primary particle. 


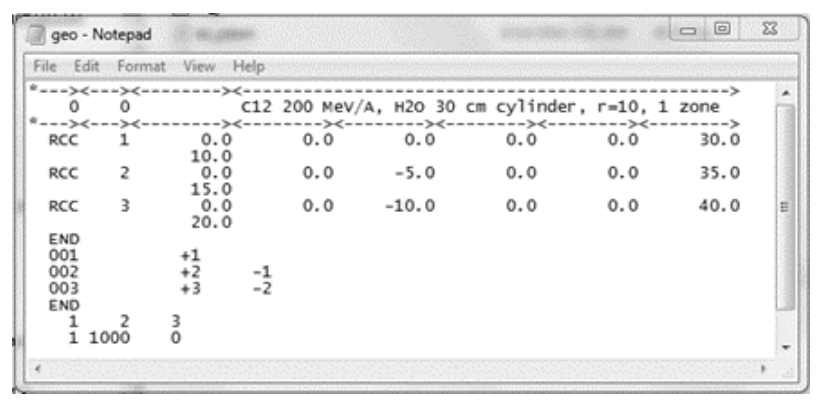

FIGURE 5. Coding data specification of target geometry.

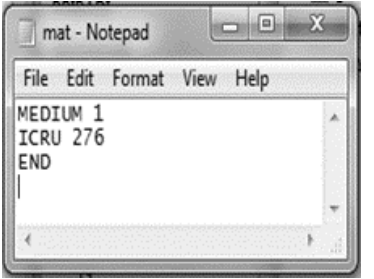

(a)

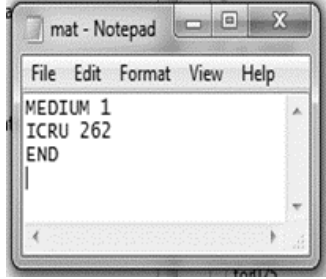

(b)
FIGURE 6. Coding of material specifications data (a) water, and (b) soft tissue.

RCC stands for Right Circular Cylinder. One side of the cylinder is described by a circle with a central point at (P1, P2, P3). The description (P4, P5, P6) holds a vector size from the center to the opposite side of the cylinder. P7 represents the radius (radius) of the cylinder (Bassler et al. 2017).

The material used in this cylindrical zone is water and soft tissue. Figure 6 shows the specification data of the shot material. Water is a material that is the default value of ICRU with the number 276, while soft tissue is a material that is the default value of ICRU with number 262. To visualize the target, SimpleGeo was used (Figure 7).

Figure 7 shows the visual result of SimpleGeo which is an interactive solid modeler for Monte Carlo simulation. The vacuum medium does not interact with the particles while the black hole medium has no trace of particles so the value of both is automatically zero.

Figure 8 explains how to assess the dose depth curve along the water target. The value of the quantity variation can be determined by the detector by using detect.dat data. This file is optional but very useful. A cylinder is set to follow the $\mathrm{Z}$ axis within the water target without requiring a match with geometry specifications in geo.dat.

The first card shows the value of the cylinder, starting from the point $(0,0,0)$ with a radius of $10 \mathrm{~cm}$, enveloped $2 \pi$ based on cylinder circumference and height of $30 \mathrm{~cm}$. The first three arguments on the next line represent 1 bin for radius, 1 bin for cylinder rotary angle and 300 bin according

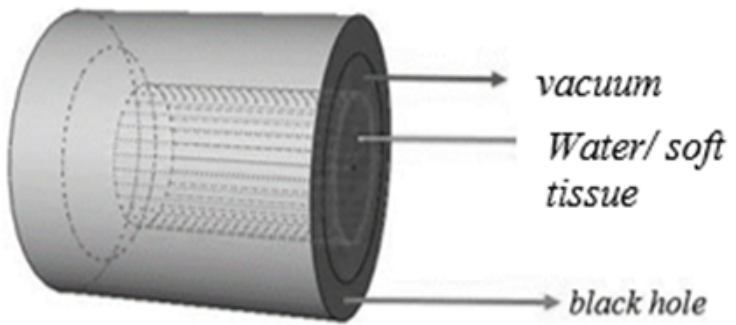

FIGURE 7. Visualizing geometry from source code example/simple using SimpleGeo. to height. The next three arguments state the energy in which the -1 particle means the energy stored by all the particles being truncated, while ex_cyl is the name of the output data.

The next two lines show connections from one bin starting at a point -5 to $5 \mathrm{~cm}$ along the $\mathrm{X}$ and $\mathrm{Y}$ axis, and 300 bin from $0 \mathrm{~cm}$ to $30 \mathrm{~cm}$ along the $Z$ axis. The results are stored in the ex_zmsh file. The next line represents a score of 100 bin from a point $-5 \mathrm{~cm}$ to $5 \mathrm{~cm}$ along the Yaxis. The next line describes a $100 \times 300$ pixel 2 -D map of the energy stored along with the inner beam of the target.

\subsection{Interaction of charged particles on BNCT}

The for024 file contains input and output data. Part of this data is copied in the output file for033. The for033 file summarizes the energy deposition in the target zone of all particles/keeping coupled with some information, the decomposition of energy decomposition according to particle generation, and the distribution of the PET isotope in the target zone.

When ionizing radiation interacts with the matter then radiation energy will decrease. Electrically charged particles will tend to interact directly. The interactions that occur are ionization and excitation events (U.S. Department of Energy 1993).

Ionization is the event of the formation of charged atoms when the atom is exposed to radiation. The outer electrons of the atoms will become free electrons (negative ions) so that the electron-deficient atoms become positive ions. Excitation is the event of higher electron transfer of energy, which changes atoms initially stable into unstable atoms (U.S. Department of Energy 1993).

The dosage calculation by SHIELD-HIT12A initially starts with the decoding of beam.dat and then the geometry code (geo.dat) and then the material code (mat.dat) is decoded. The next step is to set the stop power table using formulas according to Bethe-Bloch and Lindhard-Scharff (Equation 1).

$$
-\frac{d E}{d x}=K z^{2} \frac{Z}{A} \frac{1}{\beta^{2}}\left[\frac{1}{2} \ln \frac{2 m c^{2} \beta^{2} \gamma^{2} T_{\max }}{I^{2}}-\beta^{2}-\frac{\delta(\beta \gamma)}{2}\right]
$$

$\mathrm{A}$ is the atomic mass of the absorber. $\mathrm{Z}$ is the atomic number of the particle that occurs. $\mathrm{Z}$ is the absorbing atom number. $\mathrm{K} / \mathrm{A}$ is $0.307075 \mathrm{MeV} \mathrm{g}^{-1} \mathrm{~cm}^{2}$ for $\mathrm{A}=\mathrm{I} \mathrm{g} \mathrm{mol}^{-1}$. I is a constant ionization feature. Tmax is a transfer of the maximum energy. $\delta(\beta \gamma)$ is the effect density for the ionization of the lost energy. The Bethe-Bloch formula gives the average energy lost (power) for a heavy particle.

Figures 9 and 10 show the ionization energy distribution and recoil activity in both water and soft tissue hav-

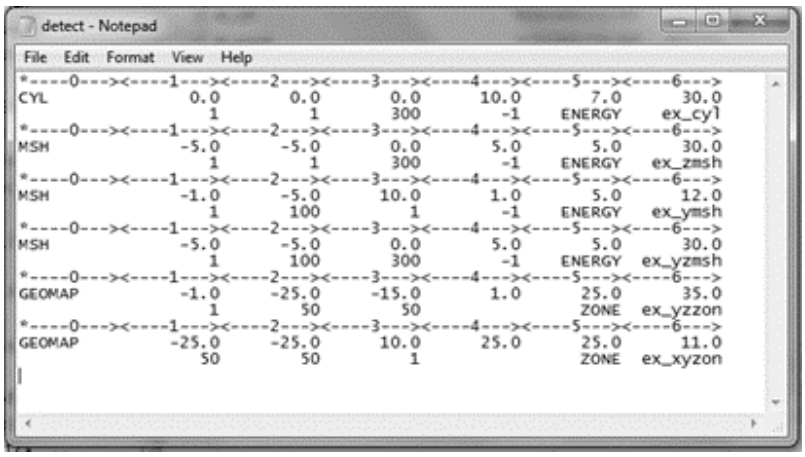

FIGURE 8. Coding additional data specifications. 


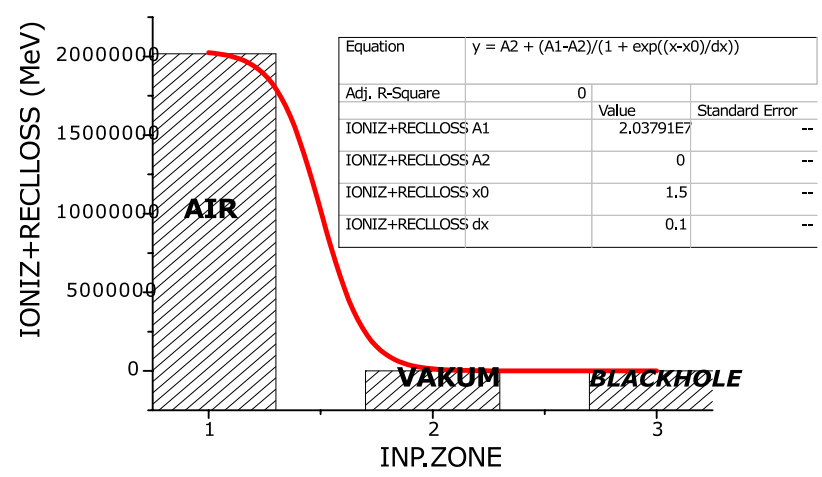

FIGURE 9. Graph of ionization energy distribution and recoil activity lost on water targets

ing significant energy depletion when reaching the vacuum medium layer. The ionization energy in the original $20379100 \mathrm{MeV}$ water becomes 0 when it reaches the vacuum zone. So, the soft tissue medium has an energy of $13030000 \mathrm{MeV}$. This is because the vacuum medium does not have a molecule that can act as an energy wave medium. When the recoil mass or the initial ion is low and the combination of the recoil material has a low stopping power, the collision between the initial recoil initial and the atom is rare. This can also be understood as a sequence of independent binary collisions between atoms. This type of cascade can be handled theoretically by using a binary collision simulation approach. In accordance with the User's Guide, the initial configuration file and the lost energy ionization fluctuations (decomposed energy) are simulated by the rapid implementation of the Gaussian distribution model. This Gaussian curve matching analysis has the least squares regression of 0 . The noise in the data may be due to measuring error, inaccuracy in the measuring instrument, or to the measured system behavior. The discussion focuses only on the distribution of ionization energy and the recoil activity lost on the material. Calculating the values of particle types such as protons, pawns, kaons, muons, protons, deuterons, tritons, He-3s, and others are not discussed even though the output data obtained the value of the particle count. The next important thing is to study the dose of photons received by each organ on the treatment of BNCT called dosimetry. The absorption dose is defined as the energy rate $(\mathrm{dE})$ absorbed by the material per unit of mass of the material (Dm). The absorbing dose was calculated using Equation 2.

$$
D=\frac{d E}{d m}
$$

The international unit of absorption dose is joule $/ \mathrm{kg}$ or gray (Gy) (BATAN 2013). Comparison of the concentration of 10B uptake by cancer cells and healthy cells is 10:1 (Erawati F et al. 2015).

In the "simple" data by taking the target case of water, the absorption doses in vitro and in vivo were calculated using the Carbon spreading geometry factor data for the known cylindrical crossover optimal dosage target obtained at concentrations of $3.232 \times 10^{-3}$ Gy dose. The carbon equivalent in water with the type of heavy core radiation of $64.64 \times 10^{-3} \mathrm{~Sv}$, and the dose of carbon equivalent in water with the type of thermal neutron radiation is $16.16 \times 10^{-3} \mathrm{~Sv}$. According to the allowable value specified by the International Commission on radiation protection (ICRP) 1966, the dose is considered unsafe because it is above the threshold of 1.5 Rem or $15 \times 10^{-3} \mathrm{~Sv}$.

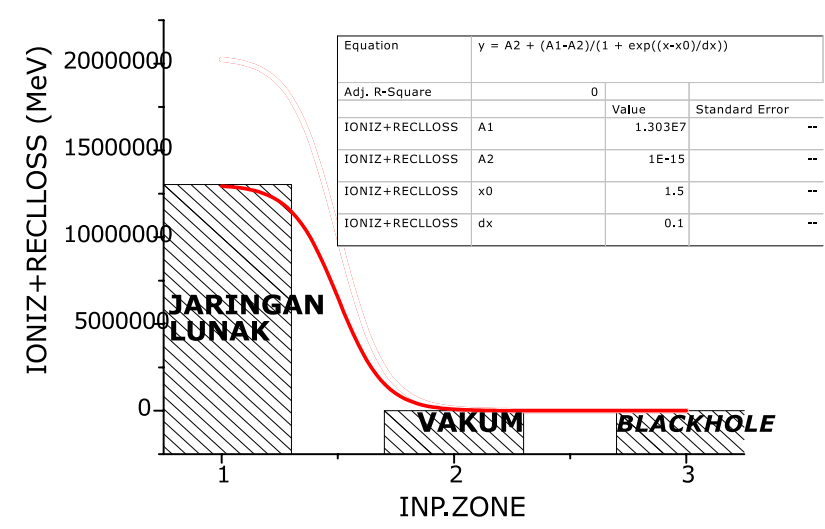

FIGURE 10. Graph of ionization energy distribution and recoil activity lost on soft tissue targets.

For case studies of soft-tissue cancer cases, the in vitro and in vivo absorption doses of tumor cells calculated using Boron spreading factor geometry data for cylindrical crosssection, also known as boron absorption dose in soft tissue (data "BNCT") was $0.208 \times 10^{-2} \mathrm{~Gy}$. The equivalent dose of boron in soft tissue with the type of heavy core radiation was $4.16 \times 10^{-2} \mathrm{~Sv}$. And the dose of boron equivalent in soft tissue with the type of thermal neutron or proton radiation was $1.04 \times 10^{-2} \mathrm{~Sv}$. The effective dose of boron in soft tissue with the type of heavy nuclear radiation is $1.248 \times 10^{-2}$ Sv. As well as the effective dose of boron in soft tissue (data "bnct") with the type of thermal neutron radiation is equal to $0.312 \times 10^{-2} \mathrm{~Sv}$. According to the permissible allowable values determined by the ICRP in 1966, this dose is safe because it falls below the threshold of 1.5 Rem or $15 \times 10^{-3} \mathrm{~Sv}$ (Rasad 2005).

In a study conducted by Zasneda (2009) in which the case of brain cancer glioblastoma multiforme was taken, the optimal dose of the tumor was obtained at a boron concentration of $22.1 \mu \mathrm{g}{ }^{10} \mathrm{~B} / \mathrm{g}$ of blood. This research can be used as a support for further research.

\section{CONCLUSIONS}

From the analysis of the data obtained, it can be concluded that the dosage in vitro and in vivo for BNCT for soft tissue irradiation has a value of $0.312 \times 10^{-2} \mathrm{~Sv}$. BNCT produces a safe effective dose and does not compromise healthy body tissue surrounding the cancer cells as it falls below the threshold of 1.5 Rem or $15 \times 10^{-3} \mathrm{~Sv}$, in accordance with the provisions of the top values permitted by the ICRP in 1966. In vitro and in vivo doses take a comparative target of water, the optimal target absorbent dose obtained at concentrations of $3.232 \times 10^{-3} \mathrm{~Gy}$. The dose of the carbon equivalent in water with the type of heavy core radiation of $64.64 \times 10^{-3} \mathrm{~Sv}$, and the dose of carbon equivalent in water with the type of thermal neutron radiation is $16.16 \times 10^{-3}$ Sv. According to the allowable value specified by the International Commission on Radiation Protection in 1966, the dose is considered unsafe because it is above the threshold of 1.5 Rem or $15 \times 10^{-3} \mathrm{~Sv}$. The use of the SHIELD-HIT 12A and SimpleGeo programs allows us to calculate radiation activity.

\section{Acknowledgements}

We would like to thank Niels Bassler, Ph.D., the Aarhus Particle Therapy Group, Aarhus University, and the Institute of Nuclear Research, RAS, Moscow, Russia, for the use of its SHIELD-HIT12A program. 


\section{REFERENCES}

Barth RF, Grecula JC, Yang W, Rotaru JH, Nawrocky M, Gupta N, Albertson BJ, Ferketich AK, Moeschberger ML, Coderre JA, Rofstad EK. 2004. Combination of boron neutron capture therapy and external beam radiotherapy for brain tumors. Int J Radiat Oncol Biol Phys. 58(1):267-277. doi:10.1016/S0360-3016(03)01613-4.

Bassler N, Luehr A, Hansen DC, Sobolevsky N. 2017. SHIELDHIT12a - user's guide.

BATAN. 2013. Radiasi [Adobe Digital Editions]. Yogyakarta: BATAN Pusdiklat eLearning.

Erawati F O, Riyatun R, Suharyana S. 2015. Modification of materials and thickness layer of radial piercing beamport (RPB) reflector on Kartini reactor for boron neutron capture therapy (BNCT). Indones J Appl Phys. 5(01):94. doi:10.13057/ijap.v5i01.270.

Matsumura A, Kumada H, Yoshioka M, Kiyanagi Y, Nakashima H. 2013. Current status of accelerator BNCT at Tsukuba and considerations for accelerator based neutron source. Tsukuba: Tsukuba University.

Rasad S. 2005. Radiologi diagnostik [Diagnostic radiology]. 2nd edition. Jakarta: Badan Penerbit FKUI.
Rasouli FS, Masoudi SF. 2012. Simulation of the BNCT of brain tumors using MNCP code: beam designing and dose evaluation. Iranian J Medi Phys. (3). doi:10.22038/ ijmp.2012.150.

Sauerwein W, Wittig A, Moss R, Nakagawa Y, editors. 2012. Neutron capture therapy: principles and applications. Berlin, Heidelberg: Springer. doi:10.1007/ 978-3-642-31334-9.

Solleh MRM, Tajuddin AA, Mohamed AA, Munem EMEA, Rabir MH, Karim JA, Yoshiaki K. 2011. Collimator and shielding design for boron neutron capture therapy (BNCT) facility at TRIGA MARK II reactor. J Nucl Relat Technol. 8(2):41-48.

US Department of Energy. 1993. DOE Fundamentals Handbook, Nuclear Physics and Reactor Theory - DOE Technical Standards Program. Washington, D.C.: U.S. Department of Energy.

Zasneda S. 2009. Perhitungan dosis pada boron neutron cancer therapy (BNCT) dengan menggunakan faktor geometri permukaan bola untuk kasus glioblastoma multiforme [master's thesis]. [Bandung]: Institut Teknologi Bandung. 